López-García, R.; Cruz-Castruita, R.M.; Morales-Corral, P.G.; Banda-Sauceda, N.C.; LagunésCarrasco, J.O. (2019). Evaluation of Bone Mineral with DEXA in Youth Soccer Players. Revista Internacional de Medicina y Ciencias de la Actividad Física y el Deporte vol. 19 (76) pp. 617-626 Http://cdeporte.rediris.es/revista/revista76/artevaluacion1098.htm

DOI: $10.15366 /$ rimcafd2019.76.004

\title{
ORIGINAL
}

\section{EVALUACIÓN DEL MINERAL ÓSEO CON LA DEXA EN FUTBOLISTAS JUVENILES}

\section{EVALUATION OF BONE MINERAL WITH DEXA IN YOUTH SOCCER PLAYERS}

López-García, R. ${ }^{\text {; }}$ Cruz-Castruita, R.M. ${ }^{2}$; Morales-Corral, P.G. ${ }^{3}$; BandaSauceda, N.C. ${ }^{4}$ y Lagunés-Carrasco, J.0. ${ }^{5}$

${ }^{1}$ Doctor en Ciencias de la Actividad Física y Deporte. Docente e investigador de la Facultad de Organización Deportiva. Universidad Autónoma de Nuevo León (México) ricardo.lopezgr@uanl.edu.mx

2 Doctora en Ciencias de Enfermería. Docente e investigador de la Facultad de Organización Deportiva. Universidad Autónoma de Nuevo León (México) rosa.cruzcst@uanl.edu.mx

${ }^{3}$ Doctor en Medicina del Deporte. Docente e investigador de la Facultad de Organización Deportiva. Universidad Autónoma de Nuevo León (México) pedro.moralescr@uanl.edu.mx

${ }^{4}$ Máster en Alto Rendimiento Deportivo. Docente de la Facultad de Salud Pública y Nutrición. Universidad Autónoma de Nuevo León (México) nancy.bandasc@uanl.edu.mx

5 Máster en Alto Rendimiento Deportivo. Docente de la Facultad de Organización Deportiva. Universidad Autónoma de Nuevo León (México) jlaguneshb23@gmail.com

CONFLICTO DE INTERÉS: En la presente investigación no existe ningún conflicto de interés.

Código UNESCO / UNESCO code: 2402 Antropología (Física) / Anthropology (Physics)

Clasificación Consejo de Europa / Council of Europe classification: 8. Cinantropometría / Kineanthropometry

Recibido 6 de febrero de 2018 Received February 6, 2018 Aceptado 7 de mayo de 2018 Accepted May 7, 2018

\section{RESUMEN}

El objetivo de este estudio fue evaluar la densidad mineral ósea (DMO) y el contenido mineral óseo $(\mathrm{CMO})$ de los segmentos corporales durante un periodo de entrenamiento de seis meses. Se evaluaron a 41 futbolistas juveniles profesionales en dos momentos, una al comienzo ( $\mathrm{Tl}$ ) y otra al final (TF) de la intervención con el 
equipo de absorciometría dual de rayos $X$ (DEXA). Se lograron aumentos significativos en la DMO en los segmentos corporales de la cadera, columna lumbar, triangulo de Ward, tronco y del cuerpo total $(\mathrm{p}<0.05)$. También se obtuvo un incremento significativo del $\mathrm{CMO}$ en la cadera, columna lumbar, pierna, tronco y costillas $(p<0.05)$. El entrenamiento de futbol fortaleció el CMO y la DMO del hueso de la extremidad inferior y de la caja torácica, con lo cual el fútbol podría ser una actividad útil para la mejorar la mineralización y fortalecimiento del hueso, para prevenir lesiones y fracturas.

PALABRAS CLAVES: Futbol, DEXA, mineral, densidad, hueso.

\section{ABSTRACT}

The objective of this study was to assess bone mineral density (BMD) and bone mineral content (BMC) of body segments for a six months training period. 41 professional youth players were evaluated in two moments, one at the beginning (TI) and another at the end (TF) of the intervention with the dual energy x-ray absorptiometry equipment (DEXA). Significant increases in BMD were achieved in the body segments of the hip, lumbar spine, ward triangle, trunk and total body $(p$ $<0.05)$. There was also a significant increase in BMC in the hip, lumbar spine, leg, trunk and ribs $(p<0.05)$. Soccer training strengthened the BMC and BMD of the lower limb bone and the rib cage, which could be a useful activity to improve bone mineralization and strengthening, to prevent injuries and fractures.

KEYWORDS: soccer, DEXA, mineral, density, bone.

\section{INTRODUCCIÓN}

El ejercicio y el deporte durante la etapa de crecimiento aceleran los niveles del mineral óseo, suministrando estímulos apropiados al hueso, logrando un fortalecimiento y un mantenimiento (Cervinka, Tittweger, Hyttinen, Felsenberg y Sievänen, 2011; Creighton, Morgan, Boardley y Brolinson, 2001; Meyer et al., 2004; Quintas, Ortega, López-Sobaler, Garrido y Requejo, 2003), para prevenir el padecimiento de fracturas en edades futuras (Christoffersen et al., 2015). Realizar actividad física en la adolescencia y en la etapa del adulto joven se pueden alcanzar el pico más alto de masa ósea (Van Langendonck et al., 2003), sin olvidar que la estructura del hueso también puede alterarse por el metabolismo de los tejidos óseos, la predisposición genética del sujeto, el comportamiento del sistema endocrino (Oh et al., 2005), control hormonal (Sun, Davies, Blair, Abe y Zaidi, 2006) y la ingesta de algunos minerales como el calcio y fosforo (Manuel Gómez, 2006).

Varios estudio con población en atletas o en personas que realizan actividad física descubrieron los diferentes efectos que pueden causar la mineralización o la remodelación ósea. Deportes donde el hueso tiene una importante carga o un alto impacto, suele tener efectos positivos en el mineral óseo (Kontulainen et al., 2001; 
Morel, Combe, Francisco y Bernard, 2001), tales como el tenis, voleibol y el fútbol. Sin embargo los deportes como el ciclismo y natación, que se practica mayormente sin ninguna carga a la masa ósea, ya sea soportar el peso corporal o tener bajo impacto sobre el hueso, no suelen tener aumentos significativos del mineral óseo en su cuerpo (Söderman, Bergström, Lorentzon, y Alfredson, 2000; Milgrom et al., 2000).

Otros deportes donde no suelen tener efectos positivos en la masa ósea son en los de resistencia como el maratón, marcha y de larga distancia (Calbet, Dorado, DiazHerrera, y Rodríguez-Rodríguez, 2001), donde por el déficit de ingesta de calorías, pueda no estar cumpliendo los requerimientos necesarios que necesita el cuerpo de algunos minerales, vitaminas y proteínas, afectando la absorción del hueso, teniendo una mineralización ósea no muy efectiva. (Vicente-Rodríguez et al., 2004)

El futbol donde aplica diferentes cambios de direcciones, saltos, caídas, arranques e inmovilizar, afecta en gran medida la parte inferior del cuerpo, debido a la fuerza de reacción en los movimientos, que hace aumentar el estímulo del hueso (Calbet et al., 2001). Se ha justificado que los futbolistas suelen tener un alto aumento en el CMO (Calbet et al., 2001; Vicente-Rodríguez et al., 2004), provocado por el trabajo físico en algunas zonas del cuerpo, ya que las células óseas en especial la formación de los osteoblastos puede comportarse diferentes en estas zonas, en comparación a las zonas no trabajadas (Khan, 2001; Scott, Khan, Duronio y Hart, 2008). Es por eso que es calificado un deporte de alto impacto y osteogénico (Calbet et al., 2001).

Nuestro objetivo de este estudio fue evaluar la DMO y el CMO de los segmentos corporales (columna, cadera, brazos, piernas, tronco, costillas y cuerpo total) así como la columna lumbar y la zona del femoral (cuello, trocánter y triangulo de Ward) durante un periodo de entrenamiento de seis meses en futbolistas juveniles, donde se les realizo dos tomas de medición, una al inicio y otra al final.

\section{MATERIAL Y MÉTODO}

Se realizó un estudio longitudinal con un total de 41 atletas de futbol juvenil de un equipo profesional (15.85 \pm 0.98 años de edad), donde se les evaluó la DMO y el CMO por medio de la DEXA, realizando una toma inicial y una al final de un periodo de entrenamiento de seis meses de duración. Los atletas firmaron una carta de consentimiento informado, para el permiso del escaneo con la DEXA. Se incluyeron en el estudio a los futbolistas que no presentaban ningún tipo de lesión física al inicio y durante el tiempo de evaluación.

\section{Antropometría}

El protocolo de las mediciones antropométricas se realizó por una persona certificada (nivel III) por la Internacional de Avances de la Cineantropometría (ISAK), con técnicas descritas en el Manual de Protocolo Internacional para la valoración 
Antropométrica (Stewart, 2010). Se citó a los futbolistas a primera hora con un ayuno de 8 horas y si haber realizado ninguna práctica deportiva, donde se les evaluó el peso con una báscula Tanita ${ }^{\circledR}$ TBF-410 $(0-200 \mathrm{~kg} \pm 0.01 \mathrm{~kg})$ y la estatura con un estadiómetro seca $213(20-205 \mathrm{~cm} \pm 5 \mathrm{~mm})$. Para después obtener el índice de masa corporal (IMC).

\section{Absorciometría dual de rayos $X(D E X A)$}

La valoración de la DMO $\left(\mathrm{g} / \mathrm{cm}^{2}\right)$ y el CMO $(\mathrm{g})$ se realizó a través del equipo de la DEXA de la marca General Electric (GE) (GE Medical Systems Ultrasound \& Primary Care Diagnostics LLC®) Radiodensitómetro óseo con software enCORE Modelo LU43616ES. Las regiones evaluadas fueron la columna, cadera, brazos, piernas, tronco, costillas, cuerpo completo, columna lumbar (L1-L4) y la zona del femoral. En los brazos y piernas se midieron ambas extremidades del lado derecho e izquierda logrando el valor medio de ambas. La columna lumbar (L1-L4) se especifica del valor medio de las columnas L1, L2, L3 y L4 de la zona lumbar. En la zona del femoral (cuello, trocánter y triángulo de Ward) se evaluó del lado derecho e izquierdo de las extremidades inferiores y se obtuvo el valor medio de ambas.

Los criterios de clasificación de la DMO fue arrojada por el software del equipo de la DEXA, en donde la Organización Mundial de la Salud (OMS), proporciona unos rango en la DMO de una puntuación $T$ mayor o igual a -1 como calcificación adecuado, entre -1 y -2.5 como clasificación osteopenia baja y un menor o igual de -2.5 como clasificación osteoporosis.

\section{Análisis estadístico.}

Se realizó un análisis estadístico con el programa del paquete SPSS en Windows versión 21.0. En la cual la estadística descriptiva determino las medias y desviaciones estándar de las variables: mediciones básicas (edad, peso, talla e IMC) y de las mediciones de la DMO y el CMO de los segmentos corporales. También se utilizó la prueba de t-student para comparar la correlación de la toma inicial a la toma final de las variables DMO y CMO de las regiones del cuerpo. Se utilizó un nivel de análisis estadístico de significancia de $p<0.05$.

\section{RESULTADOS}

La tabla 1 muestra las características de las mediciones básicas de los sujetos de estudio. En el peso corporal se encontró un aumento significativo de la TI a la TF $(p<0.05)$. En lo que se refiere al IMC también se logró un aumento significativo de la TI a la TF $(p<0.05)$, arrojando un promedio dentro del peso normal de los percentiles por edad $(5-85)$ (NCHS, 2000).

La DMO obtuvo aumentos de la TI a la TF en todos los segmentos corporales (Tabla 2), logrando incrementos significativos en la región de la cadera, en la columna 
lumbar, en el triángulo Ward, en el tronco y en el cuerpo total durante seis meses de entrenamiento $(p<0.05)$. También se muestran que todos los valores de la DMO de la Tl y de la TF se encuentran dentro de la puntuación T como nivel adecuado en la densidad del hueso (mayor o igual a -1).

En la tabla 3 se encuentra los valores del CMO donde se obtuvo una disminución de la TI a la TF de las regiones corporales del trocánter (-1.34 g) y de los brazos ($1.2 \mathrm{~g}$ ). En las demás regiones del cuerpo se lograron cambios positivos en estos seis meses de entrenamiento, logrando aumentos significativos en las regiones de la cadera, columna lumbar, piernas, tronco y en las costillas $(p<0.05)$.

\section{DISCUSIÓN}

La medición de la estructura ósea, principalmente de la DMO y en el CMO en deportistas, es de suma importancia para su rendimiento, salud y prevenir futuras lesiones. Hay disciplinas donde las exigencias de rendimiento son mayores unas que otras, o hay deportes que suele tener un alto impacto con el hueso por los movimientos mecánicos que realiza el cuerpo, que suelen tener cambios en su mineral óseo en un periodo determinado, y pueda que resulte perjudicial o beneficiario al esqueleto humano, es por eso que se debe de llevar un control del comportamiento del mineral óseo durante el periodo de entrenamiento 0 competición. Por lo que el objetivo de este estudio fue evaluar la DMO y el CMO de las regiones corporales durante un periodo de entrenamiento de seis meses en futbolistas juveniles.

Hay deportes que se consideran de bajo impacto como el ciclismo y natación por tener un efecto osteogénico negativo en el hueso, y hay deportes de alto impacto como el futbol, voleibol, gimnasia y levantamiento de pesas, donde contribuyen al desarrollo y mantenimiento en la estructura ósea (Sherk et al., 2014). Esta teoría comprueba que algunos estudios que se realizaron con nadadores (Maïmoum et al., 2004) y ciclistas (Abe et al., 2014) obtuvieron una DMO baja del cuerpo completo en comparación con estudios que se les realizo a gimnastas (Hind, Gannon, Whatley, Cooke y Truscott, 2012), voleibolistas (López-García, Hernández-Cruz, Rangel-Colmenero y García-Sánchez, 2015), levantadores de pesas (Lafforgue, 2013) y en nuestros resultados con futbolistas.

En lo que se refiere a las zonas del cuerpo de la DMO, se tiene que detallar específicamente la región evaluada debido a las diferentes cargas mecánicas y movimientos técnicos ejecutada por la práctica deportiva y que puedan afectar al esqueleto humano. En este estudio la región donde logro una mayor DMO fueron las zonas inferiores como las piernas, triángulo de Ward y el cuello del femoral. Caso contrario se dio en las extremidades superiores, donde junto a la región de las costillas, arrojaron la DMO más baja de las demás zonas evaluadas del cuerpo, estos resultados son similares a varios estudios transversales en deportes como el futbol (Morel et al., 2001; Wittich, Oliveri, Rotember y Mautalen, 2001; 
McCLANAHAN et al., 2002; Calbet et al., 2001), tenis, béisbol, basquetbol y futbol americano (McCLANAHAN et al., 2002).

Los deportes de alto impacto donde producen una gran fuerza de contacto en las zonas inferiores como el futbol (Morel et al., 2001; Fredericson et al., 2007; Hinrichs, Chae, Lehmann, Allolio y Platen, 2010; Calbet et al., 2001; El Hage, Jaber, Jacob, Moussa, y Theunynck, 2013; Silva, Goldberg, Teixeira y Dalmas, 2011) y deportes de combate (Hinrichs et al., 2010), obtuvieron una DMO en la columna lumbar y el cuello de femoral mayor que en deportes de bajo impacto como corredores (Fredericson et al., 2007), ciclismo (Hinrichs et al., 2010), triatlón (Hinrichs et al., 2010), natación (Silva et al., 2011) y tenis (Silva et al., 2011), ya que estos deportes producen movimientos de tensión muscular sin tener un impacto en el hueso. Estos resultados se ven reflejados en nuestro estudio ya que durante seis meses de entrenamientos se encontraron aumentos significativos de la columna lumbar y triangulo Ward $(p<0.05)$.

En la literatura se ha encontrado estudios longitudinales sobre la evaluación del CMO en deportes como el voleibol, basquetbol y softball (Carbuhn, Fernández, Bragg, Green, y Crouse, 2010), en el cual lograron aumentos del cuerpo total, estos resultados son muy similares a nuestro estudio donde arrojo aumentos durante seis meses de entrenamiento, pero hay que considerar que esta población tiene una edad de $15.85 \pm 0.98$ años, etapa donde todavía hay un crecimiento corporal, y eso puede aumentar en gran medida al CMO por el crecimiento y desarrollo del hueso.

\section{CONCLUSIONES}

De los hallazgos más importantes que se encontró en este estudio después de seis meses de evaluación, fue que las extremidades inferiores obtuvo aumentos significativos $(p<0.05)$, eso se debe que la práctica del futbol afecta en gran medida al esqueleto, principalmente la zona inferior debido a los diferentes movimientos mecánicos del cuerpo, como son los saltos, cambios de direcciones y la intensidad en que se practica (Calbet et al., 2001). Esto concluye que un entrenamiento a largo plazo fortalecerá la DMO de todas las regiones evaluadas, así como las partes inferiores tienen beneficios en el CMO en los futbolistas.

Tabla 1. Mediciones básicas de los sujetos de estudios de la TI a la TF.

\begin{tabular}{lcccc}
\hline & \multicolumn{4}{c}{ Mediciones básicas } \\
\cline { 2 - 5 } & T-I & T-F & Diferencia & $\mathrm{t}$ \\
\hline Estatura $(\mathrm{cm})$ & $172.02 \pm 7.48$ & $172.02 \pm 7.48$ & & \\
Peso $(\mathrm{kg})$ & $65.60 \pm 7.20$ & $66.25 \pm 7.34$ & 0.65 & $-2.078^{*}$ \\
$\mathrm{IMC}\left(\mathrm{kg} / \mathrm{m}^{2}\right)$ & $22.13 \pm 1.57$ & $22.34 \pm 1.51$ & 0.21 & $-2.087^{*}$ \\
\hline
\end{tabular}

$\mathrm{cm}$ : centímetros; kg: kilogramos; IMC: índice de masa corporal; kg/m²: kilogramos sobre metros al cuadrado; TI: Toma inicial; TF: toma final. ${ }^{*} p \leq 0.05$ 
Tabla 2. Mediciones de la DMO de los sujetos de estudio de la TI a la TF.

\begin{tabular}{lcccc}
\hline & \multicolumn{4}{c}{ DMO $\left(\mathrm{g} / \mathrm{cm}^{2}\right)$} \\
\cline { 2 - 5 } & $\mathrm{T}-\mathrm{I}$ & $\mathrm{T}-\mathrm{F}$ & Diferencia & $\mathrm{t}$ \\
\hline Columna & $1.12 \pm 0.11$ & $1.14 \pm 0.11$ & 0.02 & -1.644 \\
Cadera & $1.32 \pm 0.10$ & $1.34 \pm 0.11$ & 0.02 & $-3.180^{*}$ \\
Columna Lumbar & $1.27 \pm 0.11$ & $1.28 \pm 0.11$ & 0.01 & $-2.404^{*}$ \\
Cuello del femoral & $1.35 \pm 0.13$ & $1.48 \pm 0.97$ & 0.13 & -0.883 \\
Trocánter & $1.18 \pm 0.15$ & $1.19 \pm 0.10$ & 0.01 & -0.680 \\
Triangulo Ward & $1.33 \pm 0.15$ & $1.35 \pm 0.16$ & 0.02 & $-4.782^{*}$ \\
Brazos & $0.95 \pm 0.09$ & $1.00 \pm 0.18$ & 0.05 & -1.948 \\
Piernas & $1.48 \pm 0.09$ & $1.49 \pm 0.13$ & 0.01 & -0.703 \\
Tronco & $1.12 \pm 0.09$ & $1.16 \pm 0.14$ & 0.04 & $-2.493^{*}$ \\
Costillas & $0.86 \pm 0.08$ & $0.87 \pm 0.08$ & 0.01 & -2.730 \\
Total & $1.32 \pm 0.08$ & $1.34 \pm 0.08$ & 0.02 & $-6.635^{*}$ \\
\hline
\end{tabular}

DMO: densidad mineral óseo; $\mathrm{g} / \mathrm{cm}^{2}$ : gramos sobre centímetros al cuadrado; TI: Toma inicial; TF: toma final. ${ }^{*} p \leq 0.05$

Tabla 3. Mediciones del CMO de los sujetos de estudio de la TI a la TF.

\begin{tabular}{lcccc}
\hline & \multicolumn{4}{c}{ CMO $(\mathrm{g})$} \\
\cline { 2 - 5 } & $\mathrm{T}-\mathrm{I}$ & $\mathrm{T}-\mathrm{F}$ & Diferencia & $\mathrm{t}$ \\
\hline Columna & $176.3 \pm 36.60$ & $178.6 \pm 39.05$ & 2.3 & -1.153 \\
Cadera & $444.5 \pm 62.65$ & $455.4 \pm 60.26$ & 10.8 & $-3.746^{*}$ \\
Columna Lumbar & $74.4 \pm 14.86$ & $75.7 \pm 14.36$ & 1.2 & $-2.026^{*}$ \\
Cuello del femoral & $7.09 \pm 0.95$ & $7.09 \pm 1.39$ & 0 & -0.004 \\
Trocánter & $19.31 \pm 12.00$ & $17.97 \pm 3.36$ & -1.34 & 0.706 \\
Triangulo Ward & $4.20 \pm 0.78$ & $4.28 \pm 0.72$ & 0.08 & -1.550 \\
Brazos & $357.5 \pm 54.04$ & $356.3 \pm 76.45$ & -1.2 & 0.156 \\
Piernas & $1245 \pm 157.4$ & $1259 \pm 165.5$ & 13.9 & $-4.620^{*}$ \\
Tronco & $861.2 \pm 136.9$ & $880.3 \pm 136.3$ & 19.1 & $-4.402^{*}$ \\
Costillas & $240.3 \pm 52.35$ & $246.2 \pm 52.34$ & 5.9 & $-2.326^{*}$ \\
Total & $2947 \pm 378.1$ & $2992 \pm 382.5$ & 45.8 & -1.081 \\
\hline
\end{tabular}

CMO: contenido mineral óseo; g: gramos; TI: Toma inicial; TF: toma final. ${ }^{*} p \leq 0.05$

\section{REFERENCIAS BIBLIOGRÁFICAS}

Abe, T., Nahar, V. K., Young, K. C., Patterson, K. M., Stover, C. D., Lajza, D. G., \& Loftin, M. (2014). Skeletal muscle mass, bone mineral density, and walking performance in masters cyclists. Rejuvenation 291-296. https://doi.org/10.1089/rej.2013.1538

Calbet, J. A., Dorado, C., Diaz-Herrera, P., \& Rodriguez-Rodriguez, L. P. (2001). High femoral bone mineral content and density in male football (soccer) players. Medicine \& Science in Sports \& Exercise, 33(10), 1682-1687. https://doi.org/10.1097/00005768-200110000-00011

Carbuhn, A. F., Fernandez, T. E., Bragg, A. F., Green, J. S., \& Crouse, S. F. (2010). Sport and training influence bone and body composition in women collegiate athletes. The 
Rev.int.med.cienc.act.fís.deporte - vol. 19 - número 76 - ISSN: 1577-0354

Journal of Strength \& Conditioning Research, 24(7), 1710-1717. https://doi.org/10.1519/JSC.0b013e3181d09eb3

Cervinka, T., Rittweger, J., Hyttinen, J., Felsenberg, D., \& Sievänen, H. (2011). Anatomical sector analysis of load-bearing tibial bone structure during 90-day bed rest and 1 year recovery. Clinical physiology and functional imaging, 31(4), 249-257. https://doi.org/10.1111/j.1475-097X.2011.01009.x

Christoffersen, T., Winther, A., Nilsen, O. A., Ahmed, L. A., Furberg, A. S., Grimnes, G., \& Emaus, N. (2015). Does the frequency and intensity of physical activity in adolescence have an impact on bone? The Tromsø Study, Fit Futures. BMC sports science, medicine and rehabilitation, 7(1), 26. https://doi.org/10.1186/s13102-0150020-y

Creighton, D. L., Morgan, A. L., Boardley, D., \& Brolinson, P. G. (2001). Weight-bearing exercise and markers of bone turnover in female athletes. Journal of Applied physiology, 90(2), 565-570. https://doi.org/10.1152/jappl.2001.90.2.565

El Hage, R., Jaber, M., Jacob, C., Moussa, E., \& Theunynck, D. (2013). Lumbar spine, hip and forearm bone mineral density in a group of Lebanese professional football players. Science \& Sports, 28(4), $204-206$. https://doi.org/10.1016/i.scispo.2012.05.002

Fredericson, M., Chew, K., Ngo, J., Cleek, T., Kiratli, J., \& Cobb, K. (2007). Regional bone mineral density in male athletes: a comparison of soccer players, runners and controls. British journal of sports medicine, 41(10), 664-668. https://doi.org/10.1136/bjsm.2006.030783

Hind, K., Gannon, L., Whatley, E., Cooke, C., \& Truscott, J. (2012). Bone cross-sectional geometry in male runners, gymnasts, swimmers and non-athletic controls: a hipstructural analysis study. European journal of applied physiology, 112(2), 535-541. https://doi.org/10.1007/s00421-011-2008-y

Hinrichs, T., Chae, E. H., Lehmann, R., Allolio, B., \& Platen, P. (2010). Bone Mineral Density in Athletes of Different Disciplines: a Cross- Sectional Study. Open Sports Sciences Journal, 3, 129-133. https://doi.org/10.2174/1875399X010030100129

Khan, K. (2001). Physical activity and bone health. Human Kinetics.

Kontulainen, S., Kannus, P., Haapasalo, H., Sievänen, H., Pasanen, M., Heinonen, A., \& Vuori, I. (2001). Good maintenance of exercise-induced bone gain with decreased training of female tennis and squash players: a prospective 5-year follow-up study of young and old starters and controls. Journal of Bone and Mineral Research, 16(2), 195-201. https://doi.org/10.1359/jbmr.2001.16.2.195

Lafforgue, P. (2013). Fracturas por sobrecarga (fracturas por fatiga y fracturas por insuficiencia ósea). EMC-Aparato Locomotor, 46(3), $1-12$. https://doi.org/10.1016/S1286-935X(13)65498-2

López-García, R., Hernández-Cruz, G., Rangel-Colmenero, B., y García-Sánchez, M. (2015). Densidad Mineral Óseo en jugadores de voleibol en un periodo de entrenamiento. Revista de Investigación y Desarrollo, 1-1: 18-22.

Maimoun, L., Mariano-Goulart, D., Couret, I., Manetta, J., Peruchon, E., Micallef, J. P., \& Leroux, J. L. (2004). Effects of physical activities that induce moderate external loading on bone metabolism in male athletes. Journal of sports sciences, 22(9), 875883. https://doi.org/10.1080/02640410410001716698

Manuel Gomez, J. (2006). The role of insulin-like growth factor I components in the regulation of vitamin D. Current Pharmaceutical Biotechnology, 7(2), 125-132. https://doi.org/10.2174/138920106776597621

McCLANAHAN, B. S., Harmon-clayton, K., Ward, K. D., Klesges, R. C., Vukadinovich, C. M., \& Cantler, E. D. (2002). Side-to-side comparisons of bone mineral density in 
upper and lower limbs of collegiate athletes. Journal of strength and conditioning research, 16(4), 586. https://doi.org/10.1519/00124278-200211000-00016

Meyer, N. L., Shaw, J. M., Manore, M. M., Dolan, S. H., Subudhi, A. W., Shultz, B. B., \& Walker, J. A. (2004). Bone mineral density of Olympic-level female winter sport athletes. Medicine \& Science in Sports \& Exercise, 36(9), 1594-1601. https://doi.org/10.1249/01.MSS.0000139799.20380.DA

Milgrom, C., Finestone, A., Simkin, A., Ekenman, I., Mendelson, S., Millgram, M., \& Burr, D. (2000). In vivo strain measurements to evaluate the strengthening potential of exercises on the tibial bone. Bone \& Joint Journal, 82(4), 591-594. https://doi.org/10.1302/0301-620X.82B4.0820591

Morel, J., Combe, B., Francisco, J., \& Bernard, J. (2001). Bone mineral density of 704 amateur sportsmen involved in different physical activities. Osteoporosis International, 12(2), 152-157. https://doi.org/10.1007/s001980170148

National Center for Health Statistics (US). Division of Health Care Statistics, \& National Ambulatory Medical Care Survey (US). (2000). National Ambulatory Medical Care Survey, Summary. US Department of Health and Human Services, Public Health Service, Centers for Disease Control, National Center for Health Statistics.

Oh, K. W., Lee, W. Y., Rhee, E. J., Baek, K. H., Yoon, K. H., Kang, M. I., \& Yoo, H. J. (2005). The relationship between serum resistin, leptin, adiponectin, ghrelin levels and bone mineral density in middle-aged men. Clinical endocrinology, 63(2), 131-138. https://doi.org/10.1111/j.1365-2265.2005.02312.x

Quintas, M. E., Ortega, R. M., López-Sobaler, A. M., Garrido, G., \& Requejo, A. M. (2003). Influence of dietetic and anthropometric factors and of the type of sport practised on bone density in different groups of women. European journal of clinical nutrition, 57, S58-S62. https://doi.org/10.1038/sj.ejcn.1601817

Scott, A., Khan, K. M., Duronio, V., \& Hart, D. A. (2008). Mechanotransduction in human bone. Sports Medicine, 38(2), 139-160. https://doi.org/10.2165/00007256200838020-00004

Sherk, V. D., Barry, D. W., Villalon, K. L., Hansen, K. C., Wolfe, P., \& Kohrt, W. M. (2014). Bone loss over one year of training and competition in female cyclists. Clinical journal of sport medicine: official journal of the Canadian Academy of Sport Medicine, 24(4), 331. https://doi.org/10.1097/JSM.0000000000000050

Silva, C. C., Goldberg, T. B., Teixeira, A. S., \& Dalmas, J. C. (2011). The impact of different types of physical activity on total and regional bone mineral density in young Brazilian athletes. Journal of sports sciences, 29(3), 227-234. https://doi.org/10.1080/02640414.2010.529456

Söderman, K., Bergström, E., Lorentzon, R., \& Alfredson, H. (2000). Bone mass and muscle strength in young female soccer players. Calcified tissue international, 67(4), 297303. https://doi.org/10.1007/s002230001149

Stewart, A. D. (2010). Kinanthropometry and body composition: A natural home for threedimensional photonic scanning. Journal of Sports Sciences, 28, 455-457. https://doi.org/10.1080/02640411003661304

Sun, L. I., Davies, T. F., Blair, H. C., Abe, E., \& Zaidi, M. (2006). TSH and bone loss. Annals of the New York Academy of Sciences, 1068(1), 309-318. https://doi.org/10.1196/annals.1346.033

Van Langendonck, L., Lefevre, J., Claessens, A. L., Thomis, M., Philippaerts, R., Delvaux, K., \& Dequeker, J. (2003). Influence of participation in high-impact sports during adolescence and adulthood on bone mineral density in middle-aged men: a 27-year follow-up study. American journal of epidemiology, 158(6), 525-533. https://doi.org/10.1093/aje/kwg170 
Vicente-rodriguez, G., Ara, I., Perez-gomez, J., Serrano-sanchez, J. A., Dorado, C., \& Calbet, J. A. (2004). High femoral bone mineral density accretion in prepubertal soccer players. Medicine \& Science in Sports \& Exercise, 36(10), 1789-1795. https://doi.org/10.1249/01.MSS.0000142311.75866.D7

Wittich, A., Oliveri, M. B., Rotemberg, E., \& Mautalen, C. (2001). Body composition of professional football (soccer) players determined by dual X-ray absorptiometry. Journal of Clinical Densitometry, 4(1), 51-55. https://doi.org/10.1385/JCD:4:1:51

Número de citas totales / Total referentes: 32 (100\%)

Número de citas propias de la revista / Journal's own referentes: $0(0 \%)$

Rev.int.med.cienc.act.fís.deporte - vol. 19 - número 76 - ISSN: 1577-0354 